\title{
Size and shape dependences of the colloidal silver nanoparticles on the light sources in photo-mediated citrate reduction technique
}

\author{
S K PANDA ${ }^{1,2, *}$, S CHAKRABORTI ${ }^{2}$ and R N BASU ${ }^{2}$ \\ ${ }^{1}$ Department of Electronics, Bidhan Chandra College, Rishra, Hooghly 712248, India \\ ${ }^{2}$ Fuel Cell and Battery Division, CSIR - Central Glass and Ceramic Research Institute, Kolkata 700032, India \\ *Author for correspondence (sovan.panda@gmail.com)
}

MS received 31 July 2017; accepted 14 November 2017; published online 26 June 2018

\begin{abstract}
Colloidal silver nanoparticles (Ag NPs) with different sizes and shapes were synthesized by photo-mediated citrate reduction of aqueous $\mathrm{AgNO}_{3}$ solution under UV, sun and visible-infrared (VIS-IR) light sources. Pale yellow colour colloid was obtained under VIS-IR light exposure, whereas reddish yellow colour colloid was yielded under UV light. The colloid prepared under natural sunlight initially appeared as yellow, which turns out to be deep green after $10 \mathrm{~h}$ exposure. The colloid prepared under UV light contains fine spherical NPs with diameter ranging from 5 to $10 \mathrm{~nm}$. In addition to spherical NPs, some low-dimensional features like triangles, hexagonal platelets, nanorods were also formed under sunlight. Similar low-dimensional features including spherical particles, long chains of particles and large leaf-like platelets were grown under VIS-IR light. The colloid prepared under UV light exhibits single plasmonic absorption peak in the violet region of the visible spectra with absorption peak centred at $\sim 405 \mathrm{~nm}$. However, the colloid prepared under sun and VIS-IR light demonstrates multiple plasmonic absorption peaks related to the multi-dimensional nanoparticles. All the NPs were made of pure silver and single crystalline. The colloid prepared under VIS-IR light exhibited significant ageing with time; whereas, the colloids prepared under UV and sunlight seem very stable against ageing even after long time.
\end{abstract}

Keywords. Colloidal nanoparticles; citrate reduction; plasmonic absorption; light sources.

\section{Introduction}

Citrate reduction technique was widely used for the preparation of colloidal NPs due to its advantages to yield NPs without aggregation and mass production at low cost. It primarily involves the reduction of noble metal salts by trisodium citrate $\left(\mathrm{Na}_{3} \mathrm{C}_{6} \mathrm{H}_{5} \mathrm{O}_{7}\right)$, where citrate not only participate in reduction process, but also serves as stabilizer by surface adsorption of (citrate) $)^{-}$ions on the NP surface, which prevents them from coalescence owing to the electrostatic repulsion of negatively charged surfaces. However, pale yellow colour colloid consisting of spherical Ag NPs forms as a consequence of the chemical reduction of aqueous $\mathrm{AgNO}_{3}$ by trisodium citrate, although the colour of the colloid solely depends on the size and shape of the particles [1,2]. Huang et al [3] reported the preparation of Ag NP-based colloids with twelve distinct colours including light yellow, yellow, light orange, orange red, red, dark red, purple, purple violet, violet, blue, light blue and green, which are attributable to various sizes and shapes of the NPs prepared by chemical reduction of aqueous $\mathrm{AgNO}_{3}$, suggesting the possibility of tuning-desired optical properties. Other studies also reported the preparation of pale yellow, dark yellow, green, blue, violet and greyish colloids of $\mathrm{Ag}$ at various stages of aggregation in chemical reduction process [4-6]. Dong et al [7] prepared deep yellow and blue coloured colloids of Ag consisting of spherical NPs and triangular nanoprism, respectively, by chemical reduction technique. It was also reported that $\mathrm{Ag}_{2}^{+}, \mathrm{Ag}_{2}^{+}$(citrate), $\mathrm{Ag}_{3}^{+}$, $\mathrm{Ag}_{4}^{2+}, \mathrm{Ag}_{8}^{4+}$, etc. clusters form as main products at the early stages of reduction [8]. Among them, $\mathrm{Ag}_{4}^{2+}, \mathrm{Ag}_{8}^{4+}$ are the most abundant species, which involve in the nucleation stage. Pillai et al [8] further reported that citrate ions undergo complexation with $\mathrm{Ag}_{2}^{+}$dimers and get stabilized and their conversion to $\mathrm{Ag}_{4}^{2+}$ become slower. The citrate complex slowly grows with time and reaches an optimized size, at which stage the strong electrostatic repulsive force of citrate prevent further aggregation. Growth of the particles in the later stages occurs via Ostwald ripening method. Xia et al [9] demonstrated that $\mathrm{Ag}_{2}^{+}, \mathrm{Ag}_{4}^{2+}$ and $\mathrm{Ag}_{8}^{4+}$ clusters are responsible for the formation of spherical NPs, whereas trimetric $\mathrm{Ag}_{3}^{+}$or $\mathrm{Ag}_{3}$ clusters can serve as nuclei for growth of triangular NPs.

In citrate reduction method, particle size and agglomeration state of $\mathrm{Ag}$ NPs depend on the $\mathrm{AgNO}_{3}$ concentration, citrate concentration, reaction time, temperature and the energy supplied $[7,8,10,11]$. Therefore, an external energy source is a fundamental requirement to maintain the reaction kinetics. In general, energy is supplied in terms of heat to the solution [8-10]. However, uses of other energy sources were also reported in citrate reaction technique. Ultrasonic power, microwave dielectric heating, X-ray, $\gamma$-ray, UV and laser radiation were also used in citrate reduction technique to synthesize colloidal metal NPs [12-16]. Henglein 

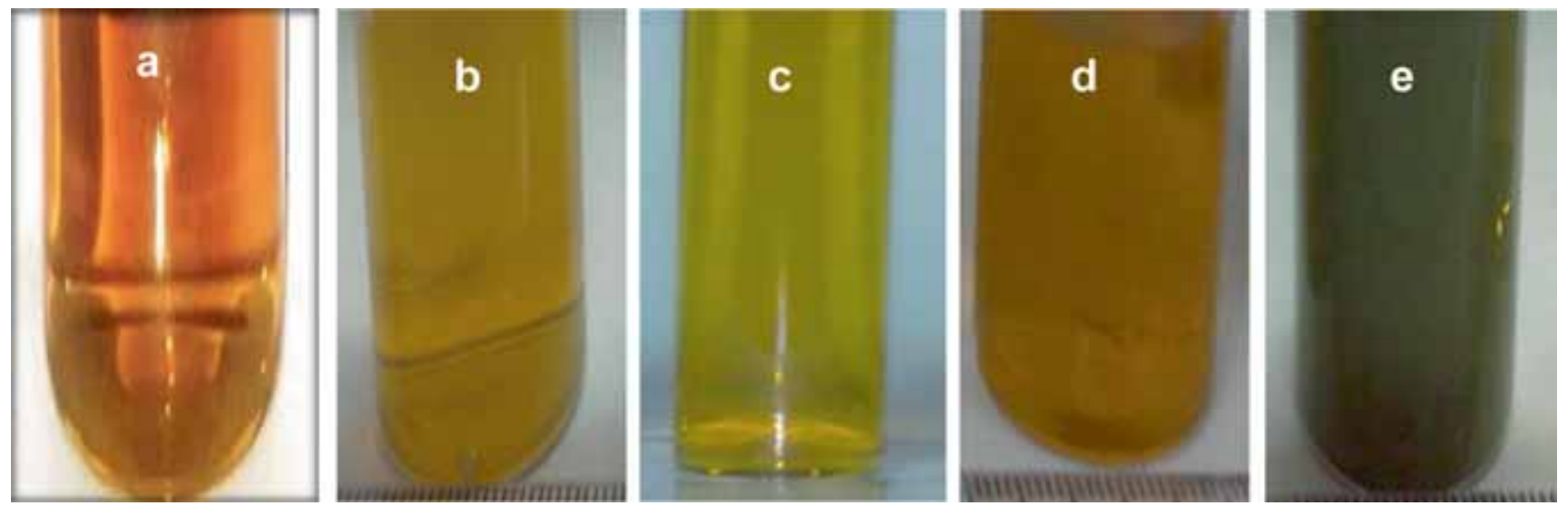

Figure 1. Optical image of the Ag NP-based colloids prepared under UV, VIS-IR light and natural sunlight: (a) UV light, (b) VIS-IR light, (c-e) natural sunlight for (c) 3 (d) 5 and (e) $10 \mathrm{~h}$ exposures.

et al [17] prepared stable $\mathrm{Ag}$ sol by $\gamma$-irradiation of a solution containing $\mathrm{AgClO}_{4}, \mathrm{~N}_{2} \mathrm{O}$, 2-propanol and citrate for various concentrations. Pillai et al [8] also prepared colloidal Ag NPs by $\gamma$-radiolysis of aqueous $\mathrm{AgNO}_{3}$ containing different sodium citrate concentrations. Ghosh et al [18] synthesized colloidal Ag NPs by reducing Ag containing salts using aqueous TX-100 under UV irradiation. Millard et al [19] studied photo-reduction of silver ions by citrate under Ar laser beam and seed growth of Ag NPs. Linnert et al [20] reported the photo-oxidation of colloidal Ag NPs in the presence of nitrous oxide. Synthesis of colloidal Ag NPs under natural sunlight was reported [21].

Herein, preparation of colloidal Ag NPs by photo-mediated citrate reduction technique under UV, VIS-IR and natural sunlight is reported and dependence of size and shape of the NPs on the energy sources used are discussed comparatively. Finally, the condition for achieving stable colloid consisting of fine, uniform, spherical and single crystalline Ag NPs is discussed.

\section{Experimental}

Colloidal Ag NPs were prepared by reducing aqueous $\mathrm{AgNO}_{3}$ solution using aqueous trisodium citrate $\left(\mathrm{C}_{6} \mathrm{H}_{5} \mathrm{Na}_{3} \mathrm{O}_{7}\right)$ in the presence of three distinct types of energy sources viz. UV, VIS-IR and natural sunlight. At first, $0.2 \mathrm{ml}$ aqueous trisodium citrate $\left(\mathrm{C}_{6} \mathrm{H}_{5} \mathrm{Na}_{3} \mathrm{O}_{7}\right)$ solution was mixed properly with $30 \mathrm{ml}$ of $0.5 \mathrm{mM}$ aqueous $\mathrm{AgNO}_{3}$ solution. For UV exposure, the mixture solution was poured in a glass Petri dish (lid open) and placed into a UV cabinet consisting of UV discharge tube (wavelength $\sim 365 \mathrm{~nm}$ and power $\sim 8 \mathrm{~mW}$ ) for 2 and $1 / 2 \mathrm{~h}$. For photo-induction under sunlight, the mixture was poured into glass culture tubes and exposed under natural sunlight continuously for 3,5 and $10 \mathrm{~h}$. Arrangement for photo reduction by VIS-IR light was similar to sunlight, but the light source in this case was a $250 \mathrm{~W}$ visible-IR-lamp, which was placed at a distance of $\sim 1 \mathrm{ft}$ from the culture tube and the exposure time was $12 \mathrm{~h}$. The as-prepared colloids were characterized by UV-VIS spectroscopy, transmission electron microscopy (TEM), energy-dispersive X-ray spectroscopy (EDS) attached to TEM. For TEM study, sample was prepared by drying a drop of colloid on the carbon-coated holed copper grid and micrographs were taken at $200 \mathrm{kV}$.

\section{Results and discussion}

Figure 1a-e shows optical images of silver colloids prepared under UV, VIS-IR light and natural sunlight. Colour change of the colloids was observed depending on the energy sources used. Reddish yellow colour colloid (figure 1a) was originated under UV light for 2 and $1 / 2 \mathrm{~h}$ exposure time, whereas pale yellow colour colloid (figure 1b) was obtained under VISIR light after $12 \mathrm{~h}$. However, both the colloids were unstable beyond the specified exposure time i.e., 2 and $1 / 2 \mathrm{~h}$ for $\mathrm{UV}$ and $12 \mathrm{~h}$ for VIS-IR light, which turned into blackish within a day due to sedimentation of agglomerated Ag particles. Therefore, exposure time for the colloids prepared under UV and VISIR light were set as 2 and $1 / 2 \mathrm{~h}$ and $12 \mathrm{~h}$, respectively, for our study. The colloid prepared under natural sunlight initially appeared as yellow colour up to $5 \mathrm{~h}$ exposure (figure 1c, d), which changed to deep green colour after $10 \mathrm{~h}$ (figure 1e). The green colour colloid was extremely stable and did not show any colour change beyond $10 \mathrm{~h}$ exposure.

UV-VIS absorption spectra of the as-prepared colloids are illustrated in figure 2 . The spectra consist of absorption peaks in the visible region of the electromagnetic spectrum, which are related to the surface plasmonic vibration caused by the oscillation of the conduction electrons in the Ag NPs with the same frequency as the incoming light. Plasmonic vibrational frequency, however, depends on the size and shape of the NPs $[2,22]$. The colloid prepared under UV light demonstrates single and intense plasmonic absorption peak centred at $\sim 405$ $\mathrm{nm}$, which is attributed to the dipole resonance, caused by the oscillation of the conduction electron at the same frequency 


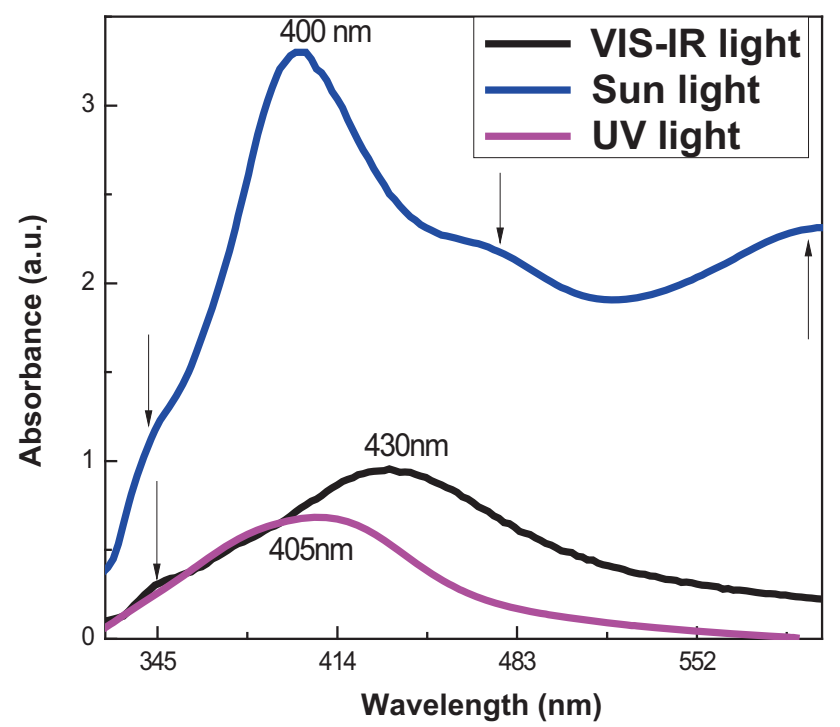

Figure 2. UV-VIS absorption spectra of the Ag NP-based colloids prepared under UV, VIS-IR light and natural sunlight.

as the incident light. Intensity of the absorbance spectra is proportional to the number density of the particles present in the colloid [23]. Position of the absorption peak centre is related to the size of the NPs present [24,25]. Smaller absorption peak position indicates formation of fine NPs in the colloid. Highly symmetric feature like spheres generally produces single resonance peak [22]. Therefore, intense single absorption peak in the spectrum indicates the presence of uniform and spherical NPs with significantly large-number density [20]. The colloid prepared under VIS-IR light demonstrates a significant red shift of the most intense absorbance peak with respect to the previous one with the peak centred at $\sim 430 \mathrm{~nm}$ and a weak shoulder at $\sim 350 \mathrm{~nm}$. Red shift of the main plasmonic absorbance peak may be due to the formation of larger-sized NPs in the colloid [8]. However, the weak shoulder appeared at $\sim 350 \mathrm{~nm}$ may indicate the presence of very fine spherical NPs together with larger NPs in the colloid or because of the quadrupole resonance caused by the nonuniform incident of light across the sphere or due to the development of low-dimensional nonspherical features in the colloid [26]. Absorption spectrum of the colloid prepared under natural sunlight seems to be completely different from others. The spectrum consists of two intense plasmonic absorbance peaks at $\sim 400$ and $\sim 600 \mathrm{~nm}$ with two shoulders at $\sim 340$ and $\sim 470 \mathrm{mn}$. Multiple resonance peaks in the spectrum originate from the other low-dimensional features apart from the spherical NPs, because of the decreased symmetry of the particles [22]. Low-symmetry features cause nonuniform distribution of electron density, which are sometimes referred as 'lightning rod' effect. It is a common tendency for the charges to be concentrated at the sharp corners of lowsymmetry features. Superior charge separation reduces the Coulombic restoring force, increasing the time required for

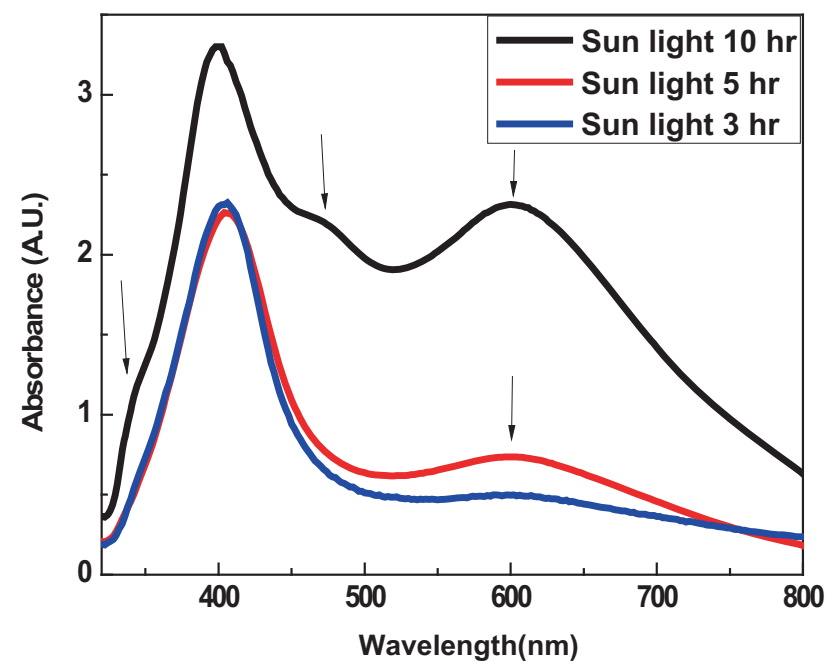

Figure 3. UV-VIS absorption spectra of the colloid prepared under sunlight for different exposure times.

each oscillation and lowering the frequency, which appears as 'red-shifting' of resonance peak compared to spherical particles in addition to the appearance of multiple peaks in the spectrum. Therefore, the green colloid may consist of lowsymmetry features together with fine Ag NPs.

Figure 3 demonstrates UV-VIS absorption spectra of the colloid prepared under sunlight for different exposure times. Absorption spectrum of the colloid prepared for $3 \mathrm{~h}$ exposure time shows an intense peak centred at $\sim 400 \mathrm{~nm}$ and a very weak and broad peak at $\sim 600 \mathrm{~nm}$ (intensity ratio between the peaks $\mathrm{I}_{400} / \mathrm{I}_{600}=4.6$ ), indicating the formation of mostly very fine and uniform spherical NPs with a few low-symmetric features in the colloid. After $5 \mathrm{~h}$ exposure time, intensity of the peak at $\sim 600 \mathrm{~nm}$ was found to be enhanced a bit, reducing the intensity ratio between the peaks $\mathrm{I}_{400} / \mathrm{I}_{600}$ to 3.0. However, the peak centre position and intensity of the absorption peak at $\sim 400 \mathrm{~nm}$ remained almost unchanged for the colloids prepared for 5 and $3 \mathrm{~h}$ exposure times. A slight broadening of the peak at $\sim 400 \mathrm{~nm}$ was observed for the colloid prepared for $5 \mathrm{~h}$ exposure time as compared to the colloid prepared for $3 \mathrm{~h}$ exposure time, which could be due to the growth of the spherical NPs with time, causing wider size distribution of the NPs. Enhancement of intensity of the peak at $\sim 600 \mathrm{~nm}$ is thought to be due to the increase in the number density of low-symmetry NPs in the colloid with increase in exposure time. A significant enhancement of the intensity and broadening of both the absorption peaks centred at $\sim 400$ and $\sim 600 \mathrm{~nm}$ was found with increase in exposure time to $10 \mathrm{~h}$ and the resulting colloid appeared as deep green colour. However, enhancement of the intensity of the peak at $\sim 600 \mathrm{~nm}$ is more as compared to the peaks at $\sim 400 \mathrm{~nm}$ reducing the intensity ratio $\mathrm{I}_{400} / \mathrm{I}_{600}$ to 1.4 . Significant reduction of $\mathrm{I}_{400} / \mathrm{I}_{600}$ of the colloid prepared for $10 \mathrm{~h}$ exposure time signifies the formation of large-number density of low-symmetry NPs as compared to spherical NPs present in the colloid. 

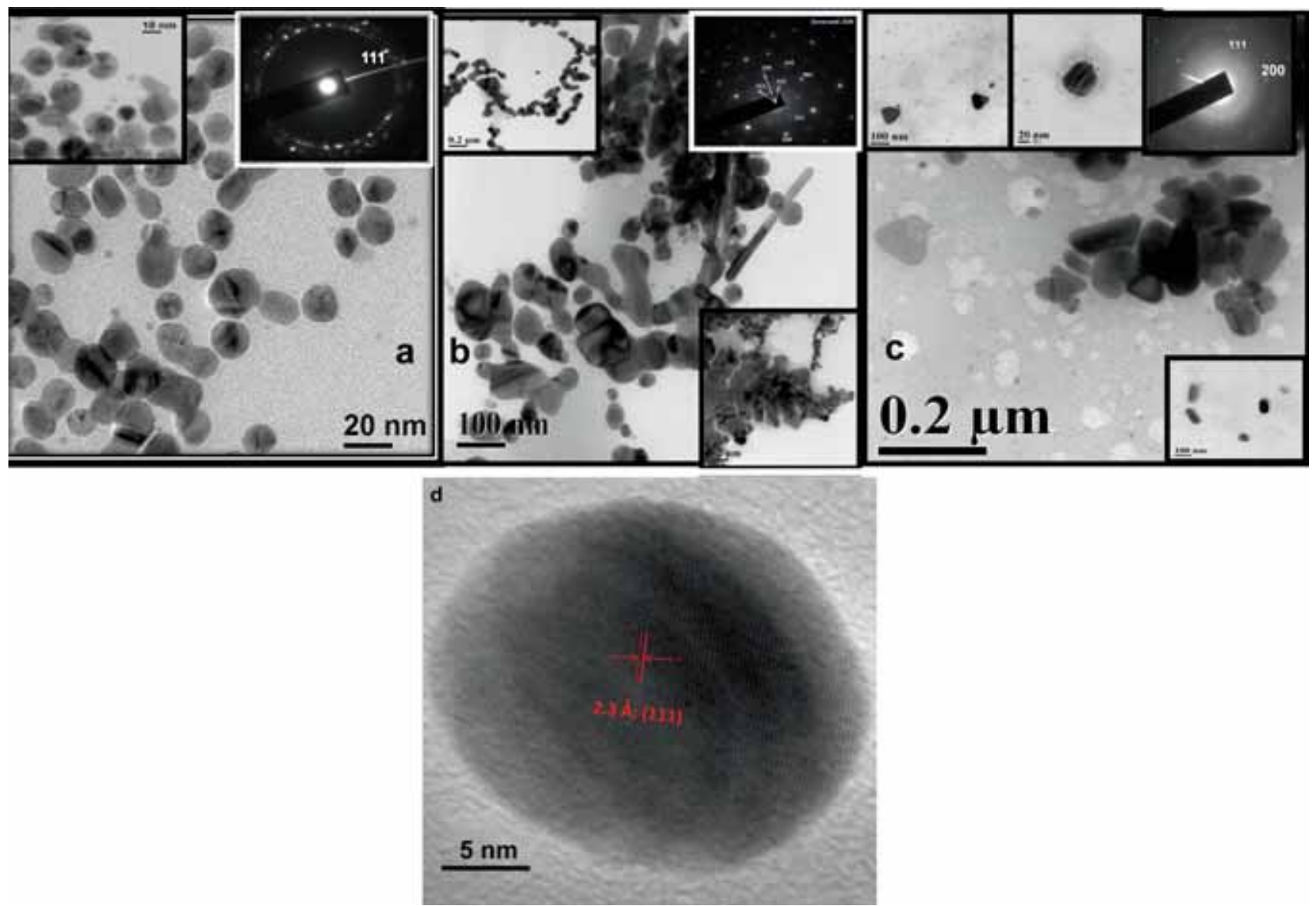

Figure 4. Bright field TEM image of the Ag NPs prepared under: (a) UV light; top left inset: TEM image of the Ag NPs with high magnification, top right inset: SAED pattern of the NPs, (b) VIS-IR light; top left inset: long chains of particles form necklace structure, top right inset: SAED pattern of the NPs, bottom right inset: large leaf-like platelet, (c) natural sunlight; top left inset: magnified image of triangular platelets and small spherical NPs, top middle inset: magnified image of hexagonal platelet, top right inset: SAED pattern of the NPs, bottom right inset: magnified image of platelets, (d) high-resolution TEM image of a single NP.

TEM study was performed to investigate the size, shape and crystallinity of the Ag NPs. Figure $4 \mathrm{a}-\mathrm{c}$ illustrates the bright field TEM image of the Ag NPs prepared under different light sources. TEM image of the NPs prepared under UV light (figure 4a and inset) confirms that the NPs are fine and spherical with almost uniform distribution of diameter ranging from 5 to $10 \mathrm{~nm}$, which is also consistent with the UV-VIS absorption study. A few elongated particles were also found in the TEM image, which could be due to the agglomeration of the spherical NPs. TEM image of figure $4 \mathrm{~b}$ is corresponding to the Ag NPs prepared under VIS-IR light, which reveals that spherical NPs with diameter ranging from 20 to $40 \mathrm{~nm}$ were grown in addition to some elongated and dumbbell-shaped NPs in the colloid. Presence of some very fine spherical NPs with diameter $\sim 2-5 \mathrm{~nm}$ was also found in the TEM image, which might be the probable reason for the appearance of small shoulder in the UV-VIS absorption spectrum. Long chains of particles (top left inset of figure $4 \mathrm{~b}$ ) and large leaf-like platelets (bottom right inset of figure $4 \mathrm{~b}$ ) were also grown in the colloid under VIS-IR light. The chain of NPs developed a necklace-like structure, which may form due to the interconnection of a number of spherical NPs with time. Shape of the NPs prepared under VIS-IR light resembles the Ag NPs of the previous report, which were prepared by $\gamma$-radiolysis of $\mathrm{AgNO}_{3}$ and sodium citrate aqueous solution [17]. TEM image of the colloid prepared under sunlight is found a bit different, which is shown in figure 3c. A large number of low-symmetry features with regular geometrical shapes including spherical, triangular, hexagonal and trapezoidal together with some one-dimensional rod-like features were formed in the colloid. Fine spherical NPs with diameter ranging from 2 to $10 \mathrm{~nm}$ coexist with the low-symmetry geometrical features. These low-symmetry geometrical features like triangular, hexagonal, trapezoidal, etc. are responsible for the multiple absorption peaks in the UV-VIS spectroscopy study.

Selected area diffraction (SAED) pattern of the NPs are shown in the inset of figure $4 a-c$, which specify that the NPs are pure crystalline silver. The diffraction spots in the SAED pattern indicate that individual NPs are single-crystalline. 


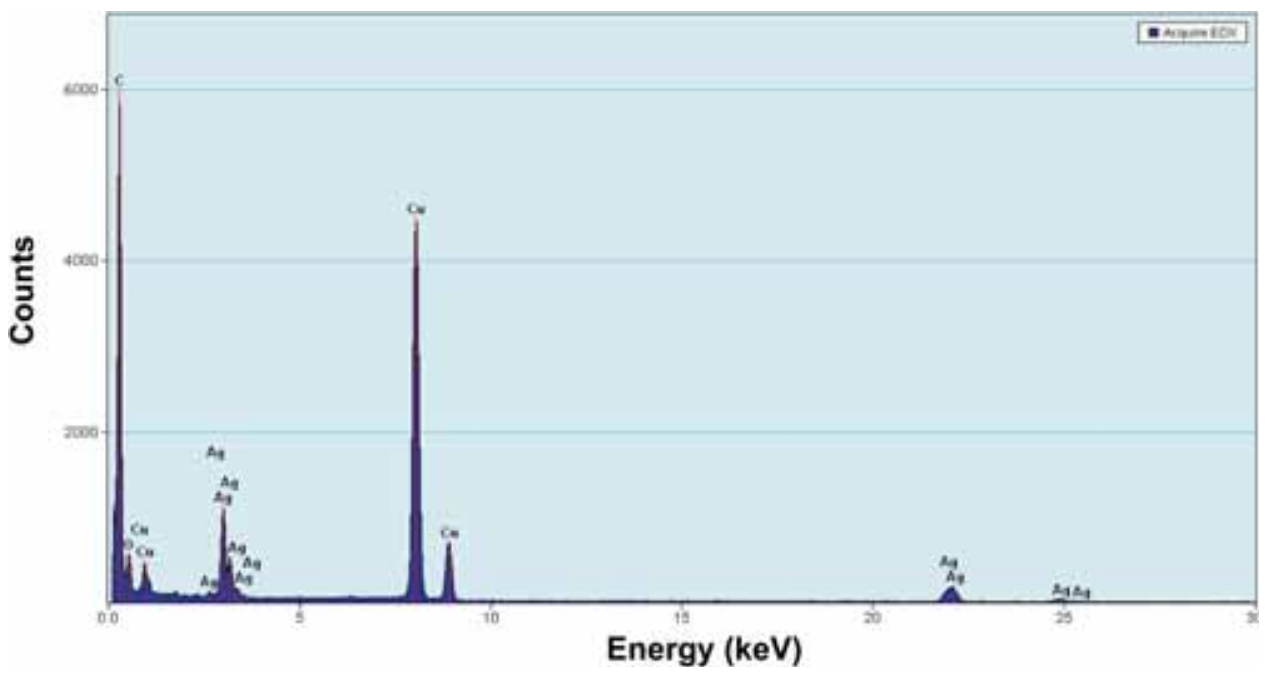

Figure 5. EDS spectrum of the NPs.

Diffraction spots are sometimes oriented along the circumference of rings, which could be due to the diffraction from a large number of very fine single-crystalline NPs, which may fit within the spot size of TEM during SAED study. Highresolution TEM image of a single NP is shown in figure 4d, where parallel lattice fringes with an inter-planer spacing of $\sim 2.3 \AA$, which is corresponding to $\mathrm{Ag}$ (111) planes of pure cubic (FCC) silver is clearly visualized throughout the NPs. Therefore, SAED pattern together with the high-resolution transmission electron microscopy (HRTEM) study proves that individual NPs are pure single-crystalline Ag.

Chemical composition of the NPs was determined using EDS spectroscopy attached to TEM system. Figure 5 shows the typical EDS spectrum of the NPs, which further indicates that NPs are made of purely Ag. However, the oxygen peak in the spectrum may appear from the oxidation of Ag NPs and/or $\mathrm{Cu}$ grid used for TEM measurement. $\mathrm{C}$ peak in the spectrum may originate from the carbon coating of the TEM grid. Another intense peak at $\sim 0.9 \mathrm{eV}$ is attributed to the $\mathrm{Cu}$ grid.

To verify the role of light source in citrate reduction technique, aqueous $\mathrm{AgNO}_{3}$ and trisodium citrate $\left(\mathrm{C}_{6} \mathrm{H}_{5} \mathrm{Na}_{3} \mathrm{O}_{7}\right)$ mixture was kept in dark for few days, but no colour change of the mixture solution was observed, which means that light plays a vital role to provide activation energy for the reaction to start. In photo-mediated citrate reduction technique, citrate molecules play important role acting as a capping ligand for the silver particles as well as a photo-reducing agent for the silver ions. The reaction that takes place in photo-mediated citrate reduction technique is as follows $[19,27,28]$ :

$$
\begin{gathered}
4 \mathrm{Ag}^{+}+\mathrm{C}_{6} \mathrm{H}_{5} \mathrm{O}_{7} \mathrm{Na}_{3}+2 \mathrm{H}_{2} \mathrm{O} \stackrel{\mathrm{h} v}{\longrightarrow} 4 \mathrm{Ag}^{0} \\
+\mathrm{C}_{6} \mathrm{H}_{5} \mathrm{O}_{7} \mathrm{H}_{3}+3 \mathrm{Na}^{+}+\mathrm{H}^{+}+\mathrm{O}_{2} \uparrow \\
\mathrm{Ag}^{+}+\mathrm{e}_{\mathrm{aq}}^{-} \rightarrow \mathrm{Ag}^{0} \rightarrow \rightarrow(\mathrm{Ag})_{n}
\end{gathered}
$$

Aqueous electrons formed during photo-oxidation of citrate and water, which reduced $\mathrm{Ag}^{+}$to $\mathrm{Ag}^{0}$. Since the aqueous electrons are strong reductants, the $\mathrm{Ag}^{+}$ions are reduced quite effectively and quickly during photo-reduction process. Citrate has three carboxylic groups and two of them would bind to the silver surface, leaving the third one normal to the surface and responsible for the colloid stabilization by electrostatic repulsion [29].

Formation of stable colloidal Ag NPs in photo-mediated citrate reduction process occurs via two steps: (a) photoreduction of $\mathrm{Ag}^{+}$ions for the formation of stable NPs (hereafter referred as seeds), (b) photo-induced growth of the seeds. Maillard et al [19] reported that growth mechanism of NPs in photo-reduction technique, which is dependent on the wavelength of the radiation source. In this study, when the spherical seeds are under UV light exposure of wavelength of $\sim 365 \mathrm{~nm}$, slightly below the spherical plasmon position, they grow uniformly with time because of isotropic light absorption, which yields spherical NPs in the colloid.

Because of some inhomogeneity in the deposited silver layer, the NPs start to grow nonuniformly, which leads to the formation of different shapes and sizes in the seed colloid and the degenerated plasmon resonance splits into transverse and longitudinal modes. The particle with the largest plasmon absorption cross-sectioned at the incident light wavelength initially, and grows fastest. The reaction accelerates for those shapes whose plasmons move into resonance with the photochemical wavelength as growth occurs.

The colloidal NPs prepared under longer wavelengths like VIS-IR and natural sunlight consist of low-symmetry features viz. triangular, hexagonal platelets, rod-like features, etc., which may originate from the inhomogeneity in growth. The longitudinal plasmon (along the longer dimension) of the elongated seed particle shifts to longer wavelength, and absorbs the longer wavelength of sunlight or IR light more strongly than the transverse mode, which shifts to shorter wavelength. If we assume that reduced atoms are deposited in the field intensity enhancement region of the surface position, the longer axis grows preferentially. The process 
Table 1. Summary of anisotropic Ag NP synthesis reports by using light/laser/ $\gamma$-ray and their comparison with the present study.

\begin{tabular}{|c|c|c|}
\hline Synthesis technique & Radiation source used & Shape of the NPs in colloid \\
\hline Citrate reduction [17] & $\gamma$-Irradiation & Spherical \\
\hline Citrate reduction $[8]$ & $\gamma$-Radiolysis & $\begin{array}{l}\text { Spherical, dumbbell-like and } \\
\text { elongated NPs }\end{array}$ \\
\hline Chemical reduction by aqueous Triton $X-100$ solution [18] & UV-irradiation & Spherical \\
\hline Citrate reduction [19] & $\begin{array}{l}\text { Ar laser-irradiation (wavelength: } \\
457 \mathrm{~nm} \text { ) }\end{array}$ & Disk-shaped Ag NPs \\
\hline Pulse laser ablation of pure $\mathrm{Ag} / \mathrm{Au}$ target in water [15] & $\begin{array}{l}\text { Switched Nd: YAG laser with } \\
\text { wavelengths: } 355 \text { and } 1064 \mathrm{~nm}\end{array}$ & Spherical \\
\hline Citrate reduction [21] & Natural sunlight & $\begin{array}{l}\text { Mixture of spherical, } \\
\text { dumbbell-like, triangular and } \\
\text { hexagonal platelets }\end{array}$ \\
\hline \multirow[t]{3}{*}{ Citrate reduction [Present study] } & UV-irradiation (365 nm) & Spherical \\
\hline & VIS-IR light & $\begin{array}{l}\text { Long chains of particles and } \\
\text { large leaf-like platelets }\end{array}$ \\
\hline & Natural sunlight & $\begin{array}{l}\text { Spherical, triangular, hexagonal, } \\
\text { trapezoidal, rod-like features }\end{array}$ \\
\hline
\end{tabular}
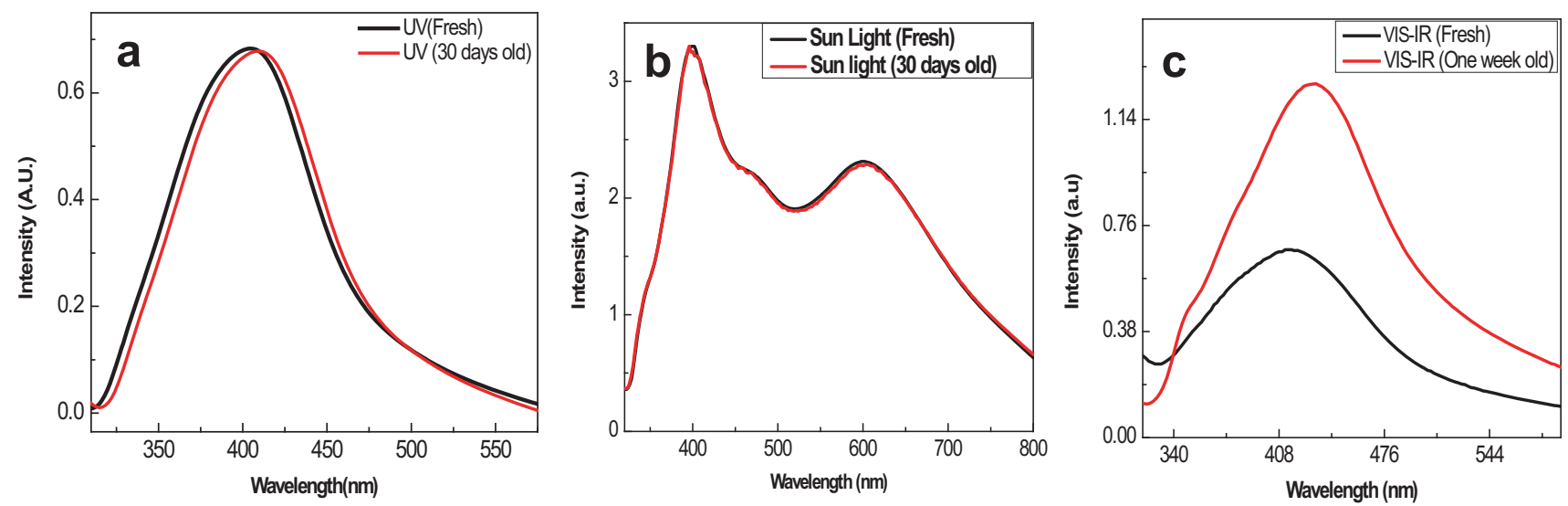

Figure 6. Ageing study by UV-VIS-NIR spectroscopy of the colloids prepared under: (a) UV light, (b) VIS-IR light and (c) sunlight.

accelerates until the longitudinal plasmon wavelength shifts beyond the irradiation wavelength resulting in the growth of low-symmetry geometrical features. Since the wavelength of VIS-IR light is long it produces long leaf-like features, because of the longer longitudinal plasmon wavelength. Solar spectrum contains a band of wavelengths from 400 to 700 $\mathrm{nm}$, which may produce low-symmetrical features with multiple dimensions. Table 1 summarizes similar type anisotropic NP synthesis reports by using light/laser/ $\gamma$-ray irradiation and their comparison with the present study.

It is also possible that photo-thermal effect promoted the shape conversion of Ag NPs in the process of the photoinduced reaction. The temperature of reaction solution was measured to be around $42^{\circ} \mathrm{C}$ when the irradiation was performed under natural sunlight or VIS-IR light. To examine the influence of heat effect from irradiation, aqueous $\mathrm{AgNO}_{3}$ and trisodium citrate $\left(\mathrm{C}_{6} \mathrm{H}_{5} \mathrm{Na}_{3} \mathrm{O}_{7}\right)$ mixture was kept wrapped with aluminium foil to eliminate the light effect under sunlight and VIS-IR light for $10 \mathrm{~h}$ and no colour change was observed. Therefore, thermal energy from the light sources was not sufficient for the chemical reduction process in this study.

Ageing study of the colloids was performed by UV-VIS spectroscopy measurement after few weeks. Figure $6 a-c$ shows a comparative study of UV-VIS spectra between the fresh and few weeks-old colloidal NPs. The absorption spectrum of the colloid prepared under sunlight does not show any change even after a month, which indicates that the colloid is extremely stable. A negligible red shift of the absorption peak was observed in the colloid prepared under UV-light even after 30 days, indicating that the colloid is a stable one. In contrast, significant increase of intensity and red shifting of the absorption peak by $\sim 13 \mathrm{~nm}$ was found after only a week of the colloid prepared under VIS-IR light. Red shift of the absorption peak of the colloid occurs due to the growth of the particles with time.

In case of the colloid prepared under UV light and sunlight, a large number of nuclei were formed at the beginning. 
The nuclei grew by joining with $\mathrm{Ag}^{+}$ion further to form the $\mathrm{AgNO}_{3}$ solution in presence of light source. Then, the NPs got surrounded by the citrate ions, which protected them from agglomeration. However, NPs can grow further with time, if there are any unreacted $\mathrm{Ag}^{+}$ions in the solution that join with the NPs even in the absence of the light source. It is probable that the colloid became deployed of $\mathrm{Ag}^{+}$ions, further growth of the NPs with time was not possible and hence, the stability of the colloid. In case of the colloid prepared under VIS-IR light, some unreacted $\mathrm{Ag}^{+}$ions may remain in the solution, which join with the NPs even in the absence of the light source. As a result, particle size may increase continuously with time, which is termed as ageing of the NPs.

\section{Conclusions}

In photo-mediated citrate reduction technique, energy source influences the size and shape of the Ag NPs. Colour of the colloid depends on the size and shape of the NPs present in the colloid. The colloid prepared under UV light contains fine spherical NPs with diameter ranging from 5 to $10 \mathrm{~nm}$. In addition to spherical NPs, some low-dimensional features like triangles, hexagonal platelets and nanorods were also formed under sunlight. Similar low-dimensional features including spherical particles, long chains of particles and large leaf-like platelets were grown under VIS-IR light. The colloid prepared under UV light exhibits single plasmonic absorption in the violet region of the visible spectra with absorption peak centred at $405 \mathrm{~nm}$. Whereas, the colloid prepared under sun and VIS-IR light demonstrates multiple plasmonic absorption peaks related to the multi-dimensional NPs. All the NPs are made of cubic single-crystalline silver. The colloid prepared under VIS-IR light exhibits significant ageing with time; whereas the colloid prepared under UV and sunlight seems very stable against ageing even after long time. UV light source produces stable, fine, uniform, spherical and single-crystalline Ag NPs as compared to other energy sources used in this study.

\section{Acknowledgements}

We acknowledge financial support of DST-INSPIRE Faculty Project, DST, New Delhi, India (Award No. IFA 12-ENG17, 2012) and active support of Director, CSIR-CGCRI and Principal, Bidhan Chandra College, Rishra, for this work.

\section{References}

[1] Lee P C and Meisel D 1984 J. Phys. Chem. 863391

[2] Gonzalez A L, Noguez C, Beranek J and Barnard A S 2014 J. Phys. Chem. C 1189128

[3] Huang T and Xu X-H N 2010 J. Mater. Chem. 209867

[4] Haes A J and Van Duyne R P 2004 Anal. Bioanal. Chem. 379 920

[5] Raja M A, Kanwal Z, Rauf A, Sabri A N, Riaz S and Naseem S 2016 Nanomaterials 674

[6] Solomon S D, Bahadory M, Jeyarajasingam A V, Rutkowsky S A, Boritz C and Mulfinger L 2007 J. Chem. Edu. 84322

[7] Dong X, Ji X, Wu H, Zhao L, Li J and Yang W 2009 J. Phys. Chem. C 1136573

[8] Pillai Z S and Kamat P V 2004 J. Phys. Chem. B 108945

[9] Xiong Y, Washio I, Chen J, Sadilek M and Xia Y 2007 Angew. Chem. Int. Ed. 464917

[10] Van Hoonacker A and Englebienne P 2006 Curr. Nanosci. 2 359

[11] Jiang X C, Chen W M, Chen C Y, Xiong S X and Yu A B 2011 Nanoscale Res. Lett. 632

[12] Gorham J M, MacCuspie R I, Klein K L, Fairbrother D H and Holbrook R D 2012 J. Nanopart. Res. 141139

[13] Ahern A M and Garrell R L 1987 Anal. Chem. 592813

[14] Patel K, Kapoor S, Dave D P and Mukherjee T 2005 J. Chem. Sci. 11753

[15] Vinod M and Gopchandran K G 2014 Prog. Nat. Sci. Mater. Int. 24569

[16] Chen H-J and Wen D 2011 Nanoscale Res. Lett. 6198

[17] Henglein A and Giersig M 1999 J. Phys. Chem. B 1039533

[18] Ghosh S K, Kundu S, Mandal M, Nath S and Pal T 2003 J. Nanopart. Res. 5577

[19] Maillard M, Huang P and Brus L 2003 Nano Lett. 31611

[20] Linnert T, Mulvaney P and Henglein A 1991 Ber. Bunsenges. Phys. Chem. 95838

[21] Tang B, Sun L, Li J, Zhang M and Wang X 2015 Chem. Eng. J. 26099

[22] Noguez C 2007 J. Phys. Chem. C 1113806

[23] Kelly K L, Coronado E, Zhao L L and Schatz G C 2003 J. Phys. Chem. B 107668

[24] Rai A, Singh A, Ahmad A and Sastry M 2006 Langmuir 22 736

[25] Wiley B J, Im S H, Li Z Y, McLellan J, Siekkinen A and Xia Y 2006 J. Phys. Chem. B 11015666

[26] Cobley C M, Skrabalak S E, Campbell D J and Xia Y 2009 Plasmonics 4171

[27] Silva E I, Rivas J, Leon L M, Isidro M A and Lopez Q 2007 J. Non-Cryst. Solids 353829

[28] Hangxun X and Kenneth S S 2010 Adv. Mater. 221078

[29] Munro C H, Smith W E, Garner M, Clarkson J and White P C 1995 Langmuir 113712 